\title{
Observational Evidence for Small-Scale Mixture of Weak and Strong Fields in the Quiet Sun
}

\author{
H. Socas-Navarro \\ High Altitude Observatory, NCAR, 3450 Mitchell Lane, Boulder, CO 80307-3000, USA \\ navarro@ucar.edu \\ B.W. Lites \\ High Altitude Observatory, NCAR, 3450 Mitchell Lane, Boulder, CO 80307-3000, USA \\ lites@ucar.edu
}

\begin{abstract}
Three different maps of the quiet Sun, observed with the Advanced Stokes Polarimeter (ASP) and the Diffraction-Limited Stokes Polarimeter (DLSP), show evidence of strong $(\simeq 1700 \mathrm{G})$ and weak $(<500 \mathrm{G})$ fields coexisting within the resolution element both at network and internetwork locations. The angular resolution of the observations is of $1^{\prime \prime}(\mathrm{ASP})$ and $0 .{ }^{\prime \prime} 6$ (DLSP), respectively. Even at the higher DLSP resolution, a significant fraction of the network magnetic patches harbor a mixture of strong and weak fields. Internetwork elements that exhibit $\mathrm{kG}$ fields when analyzed with a single-component atmosphere are also shown to harbor considerable amounts of weak fields. Only those patches for which a single-component analysis yields weak fields do not show this mixture of field strengths. Finally, there is a larger fractional area of weak fields in the convective upflows than in the downflows.
\end{abstract}

Subject headings: line: profiles - Sun: atmosphere - Sun: magnetic fields - Sun: photosphere

\footnotetext{
${ }^{1}$ The National Center for Atmospheric Research (NCAR) is sponsored by the National Science Foundation.
} 


\section{Introduction}

Our understanding of quiet Sun magnetic fields is evolving at a very rapid pace. The classical picture of the quiet Sun is based on a sprinkling of kilo-Gauss (kG) fluxtubelike structures of sub-arcsecond sizes (unresolved in the observations) forming a magnetic network at the boundaries of the supergranular cells. The internetwork (cell interiors) would be almost devoid of flux, exhibiting only sparse weak turbulent flux concentrations. As new observations became available with improved sensitivity and spatial resolution, the internetwork region started to gain importance. Strong $\mathrm{kG}$ fields were also found in many internetwork locations and the amount of magnetic flux and energy detected has increased as the instrumentation improved (Keller et al. 1994; Lin 1995; Grossmann-Doerth et al. 1996; Lin \& Rimmele 1999; Sánchez Almeida \& Lites 2000; Lites 2002; Socas-Navarro \& Sánchez Almeida 2002; Khomenko et al. 2003; Domínguez Cerdeña et al. 2003a; Domínguez Cerdeña et al. 2003b; Socas-Navarro et al. 2004; Lites \& Socas-Navarro 2004).

At the same time, the concept of organized magnetic structures (e.g., fluxtubes) as the building blocks for quiet Sun fields seems to be losing some ground, at least outside of the network. Recent numerical simulations (Cattaneo 1999; Emonet \& Cattaneo 2001; Sánchez Almeida et al. 2003; Schüssler 2003; Stein \& Nordlund 2002) reveal a more disorganized, almost chaotic, scenario with the field being dragged around by turbulent convective motions. Another important element in this picture is the discrepancy in the distribution of internetwork fields as seen in visible and infrared observations. Infrared data (Lin 1995; Khomenko et al. 2003) show a predominance of sub-kG fields, with a distribution that peaks around $350 \mathrm{G}$. On the other hand, authors working with visible observations (e.g., Socas-Navarro \& Sánchez Almeida 2002) obtain that most of these fields are of kG strength. Socas-Navarro \& Sánchez Almeida (2003) proposed that this discrepancy is a natural consequence of unresolved small-scale inhomogeneities of the field. If one has a mixture of weak and strong fields coexisting in the resolution element, visible and infrared observations tend to emphasize different parts of the distribution.

In a recent paper, Socas-Navarro (2004b) (see also Socas-Navarro 2004a) showed that the visible Fe I lines at $6302 \AA$ exhibit some sensitivity to unresolved field strength inhomogeneities. In the present work we make use of this property to seek evidence that mixed strengths indeed occur in the quiet Sun. As we discuss below, we are able to detect a rather large number of such mixed strengths. Our results support the "disorganized" picture of the

quiet Sun fields discussed above, not only in the internetwork but also to some extent in the magnetic network. 


\section{Observations and analysis}

The datasets analyzed in this paper come from two different instruments. The Advanced Stokes Polarimeter (ASP) is a spectro-polarimeter for the Dunn Solar Telescope (DST) at the Sacramento Peak observatory (Sunspot, NM, USA), operated by the National Solar Observatory. We used the map observed by Lites (1996) on Sep 29, 1994 (hereafter referred to as Map 1). This map has a spatial resolution of $\simeq 1^{\prime \prime}$ and a spectral resolution of $\simeq 30 \mathrm{~m} \AA$. The spectral lines observed are the pair of Fe I lines in the $6302 \AA$ region.

The other instrument employed is the Diffraction-Limited Stokes Polarimeter (DLSP), which is operated at the same telescope. The DLSP is a new instrument that has been designed specifically to take advantage of the new adaptive optics (AO) system at the DST in order to achieve very high angular resolution. It has been optimized for the routine observation of the $6302 \AA$ spectral region.

Lites \& Socas-Navarro (2004) obtained what can be considered very high-resolution Stokes observations of the quiet Sun, of approximately 0." 6 . This figure, alongside with the 0."5 reached by Domínguez Cerdeña et al. (2003a), represent the highest resolution spectropolarimetric observations of quiet Sun fields made thus far. In our analysis here we consider the best two maps observed by Lites \& Socas-Navarro (2004), namely the ones observed on Sep 14 and Sep 162003 (hereafter Map 2 and Map 3, respectively).

\subsection{One-component analysis}

We first conducted a one-component (hereafter 1C) analysis, assuming that we have one field strength $(B)$ that occupies a certain area filling factor $(\alpha)$ of the spatial pixel. Instead of performing an iterative least-squares fit to the observations, we chose to do forward modeling from a large number of models. In this manner we make sure that the entire model space is probed and that the absolute minimum of the $\chi^{2}$ merit function is found. We start with two models for the thermodynamic parameters (temperature, gas pressure, micro- and macroturbulence and line-of-sight velocity) of the quiet Sun, representing a granule and an intergranular lane. The models were obtained from the inversion of average Stokes I profiles. The Stokes $\mathrm{V}$ profiles were then synthesized for many values of the magnetic field strength (ranging from $B=300$ to $B=2000 \mathrm{G}$ ) and a global velocity offset (from $v=-2.5$ to

$v=2.5 \mathrm{~km} \mathrm{~s}^{-1}$ ). The calculations were performed with the code LILIA (Socas-Navarro 2001), assuming LTE and hydrostatic equilibrium.

Depending on the continuum intensity of the observations, we used the granule or lane models or a suitable linear combination of both. In the case of the quiet Sun this is approach 
is valid because the thermodynamical properties of the atmosphere experience only relatively small variations across the map. Fig 1 shows the fits obtained with this method to intensity profiles from four randomly-chosen locations. Reasonably good fits are obtained for the entire dataset analyzed, which justifies the approximation used for the thermodynamics of the atmosphere.

The synthetic Stokes V profiles $\left(V^{\text {syn }}\right)$ were multiplied by a filling factor $\alpha=A\left(V^{\text {obs }}\right) / A\left(V^{\text {syn }}\right)$ (where $A$ denotes the amplitude of a given profile) and then compared one by one to the observed profile $\left(V^{o b s}\right)$ at each spatial position of the maps. The sign of the synthetic profile is chosen to match the polarity of $V^{o b s}$. The values of $B, v$ and $\alpha$ that yield the best fit to $V^{o b s}$ are taken as representative of the conditions at the spatial location under consideration. This process is repeated for every pixel in every map. The spatial distributions of $\alpha$ and $B$ thus obtained are shown if Figs 2 and 3 (upper panels).

It is important to note that the $6302 \AA$ lines are in the weak field regime (i.e., the regime in which the Zeeman splitting is much smaller than the Doppler width) for field strengths lower than $\simeq 500 \mathrm{G}$. In the weak field regime the shape of the Stokes V profiles is independent of the magnetic field. The profiles are simply scaled with the value of the field. Thus, it is not possible to disentangle the effects of the filling factor from the field strength. In other

words, any value of the field weaker than $500 \mathrm{G}$ will result in exactly the same $\chi^{2}$. Therefore, the reader must keep in mind that any fields below $500 \mathrm{G}$ depicted in the figures might be actually weaker (with correspondingly larger filling factors).

\subsection{Two-component analysis}

Socas-Navarro (2004b) suggested that the Fe I lines at $6302 \AA$ exhibit some sensitivity to the presence of two magnetic strength components. When the two field strengths are at least as far apart as $500 \mathrm{G}$ and $1700 \mathrm{G}$, their response functions are sufficiently decoupled that the relative filling factors of these two components can be inferred with an uncertainty of 0.10 or less.

As a further step in our study, we carried out a two-component (2C) analysis of the observations. Considering the arbitrariness in the strength of the weak component we chose a value of $300 \mathrm{G}$, which is close to the peak of the distribution obtained from $1 \mathrm{C}$ inversions of infrared internetwork observations (Khomenko et al. 2003). Thus, we (arbitrarily) fixed the strengths of the weak and strong components to $300 \mathrm{G}$ and $1700 \mathrm{G}$, respectively. The problem now is to find the filling factors $\alpha_{w}$ and $\alpha_{s}$. Let us define these filling factors relative to the magnetic element, so that $\alpha_{w}+\alpha_{s}=1$. The total magnetic filling factor 

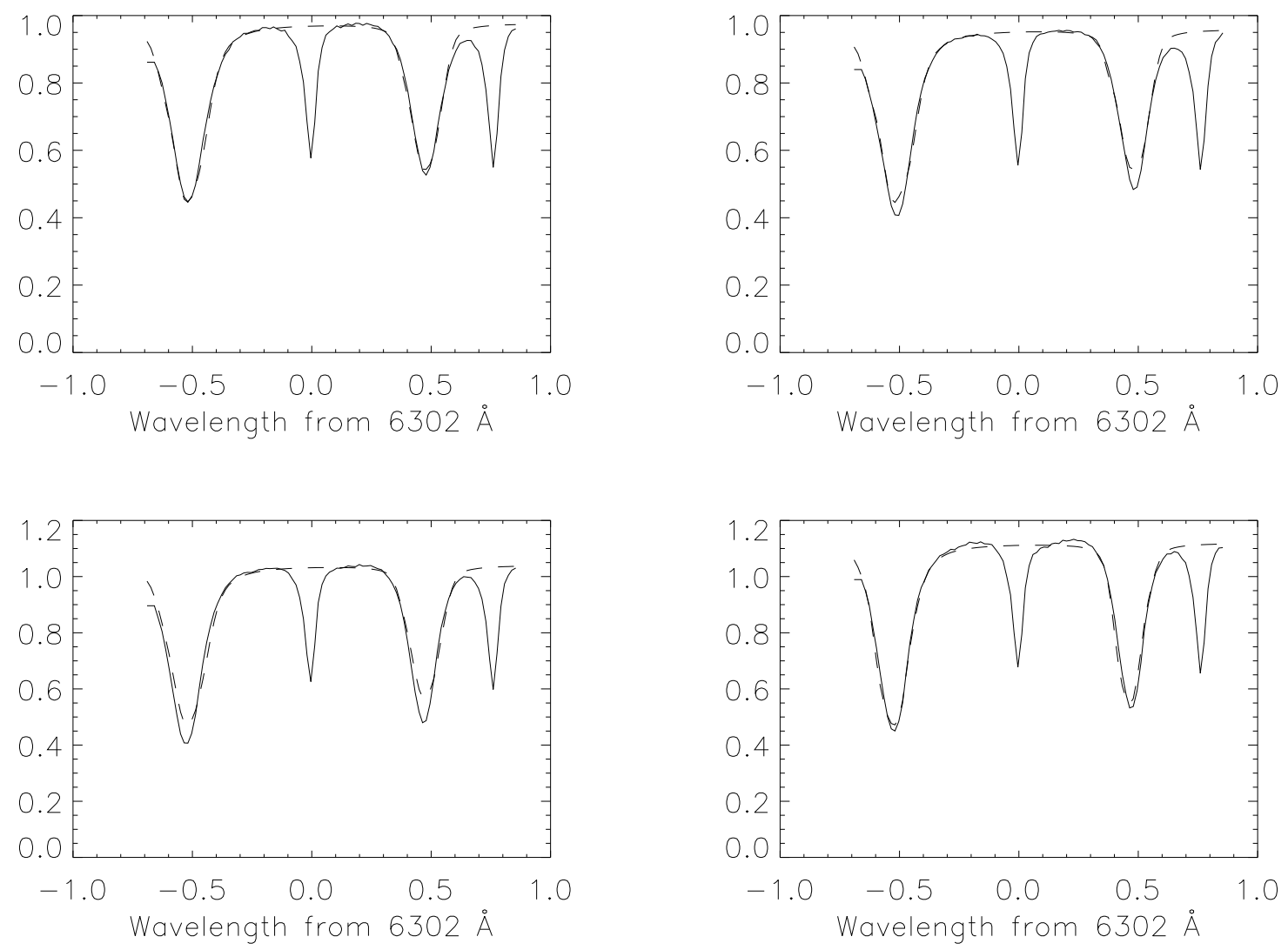

Fig. 1.- Fits (dashed) to observed (solid) intensity profiles corresponding to (x,y) coordinates $(20,60),(50,90),(80,120)$ and $(110,150)$ of Map 2. 
in the observed pixel is still $\alpha$. The filling factors of the two components relative to the resolution element are then $\alpha \alpha_{w}$ and $\alpha \alpha_{s}$. This convention may seem somewhat confusing at first, but it is useful for the discussion in $\S 3$ below.

The synthetic profiles $V^{s y n}$ for the $2 \mathrm{C}$ case were calculated in the following manner. As before, we start with the a model for the atmospheric thermodynamics which depends on the observed continuum intensity. We then synthesized the profiles $V^{w}$ and $V^{s}$ for 300 and $1700 \mathrm{G}$, respectively. At each spatial location we compared the profiles $V^{\text {obs }}$ and $\alpha V^{\text {syn }}=$ $\alpha\left(\alpha_{w} V^{w}+\alpha_{s} V^{s}\right)$ (where, again, $\left.\alpha=A\left(V^{o b s}\right) / A\left(V^{s y n}\right)\right)$. The combinations of $\alpha, \alpha_{w}, \alpha_{s}$ and $v$ that lead to the best fit of the observations are selected. Notice that only two of these parameters, $v$ and either $\alpha_{s}$ or $\alpha_{w}$, are independent.

In order to ensure that the detection of mixed field strengths has significance, we rewarded solutions with either $\alpha_{w}=0$ or $\alpha_{s}=0$. The $\chi^{2}$ corresponding to these solutions is reduced by $10 \%$. This conservative approach makes the procedure "prefer" one-component solutions. It is also important to point out that, while we are not adding any degrees of freedom when going from $1 \mathrm{C}$ (above) to $2 \mathrm{C}$, the $\chi^{2}$ is smaller in the $2 \mathrm{C}$ analysis virtually everywhere. These arguments (alongside with those in $\S 4$ ) give us confidence in the results reported below.

\section{Results}

Figs 2 and 3 show the spatial distribution of $\alpha$ (upper left), $B$ (upper right), $\alpha_{s}$ (lower left) and $\alpha_{w}$ (lower right). The $1 \mathrm{C}$ analysis results in strong $\mathrm{kG}$ fields in most spatial pixels inverted (i.e., those exhibiting significant polarization signal), with the exceptions of very few weak field patches in the network and some weak field elements in the internetwork. This is consistent with previous observations published in the literature based on visible lines, in which the distribution of fields peaks around $1.5 \mathrm{kG}$ (see references in $\S 1$ ).

The lower panels of the figures clearly show the presence of mixed-strength pixels both in network and internetwork locations. Let us start by discussing the results for the internetwork. In this region, visible and infrared observations (always using 1C analyses) have led to disparate conclusions, with the infrared lines showing a much larger fraction of weaker fields. According to Socas-Navarro \& Sánchez Almeida (2003), this can be explained by a small-scale mixture of weak and strong fields beyond the spatial resolution. They showed that, when such mixture exists, a $1 \mathrm{C}$ inversion of the visible lines is biased towards the stronger fields. Therefore, we would expect to have mixed field strengths in those spatial pixels where the $1 \mathrm{C}$ analysis results in strong fields. Those with weak fields, on the other 
hand, are probably rather homogeneous. This is exactly what we find in the analysis of our internetwork profiles, as seen in Figs 2 and 3, as well as in Table 1. We did not find any mixed strengths in pixels with $\alpha<0.1$ and $B<500 \mathrm{G}$.

Let us now turn to the network. The relatively strong flux concentrations in network patches have been traditionally associated with strong $\mathrm{kG}$ fields (see references in $\S 1$ ). While our $1 \mathrm{C}$ analysis agrees with this assessment, the $2 \mathrm{C}$ analysis reveals the presence of a significant amount of weak fields mixed at small scales. The mixed strengths occur all over the network patches and not only around their perimeter. Table 1 lists some properties of the network elements, including the percentage of pixels showing mixed strengths. Finally, we find (not shown in the table) that the fractional area occupied by weak fields is larger in the convective upflows than in the downflows (as one would expect). 

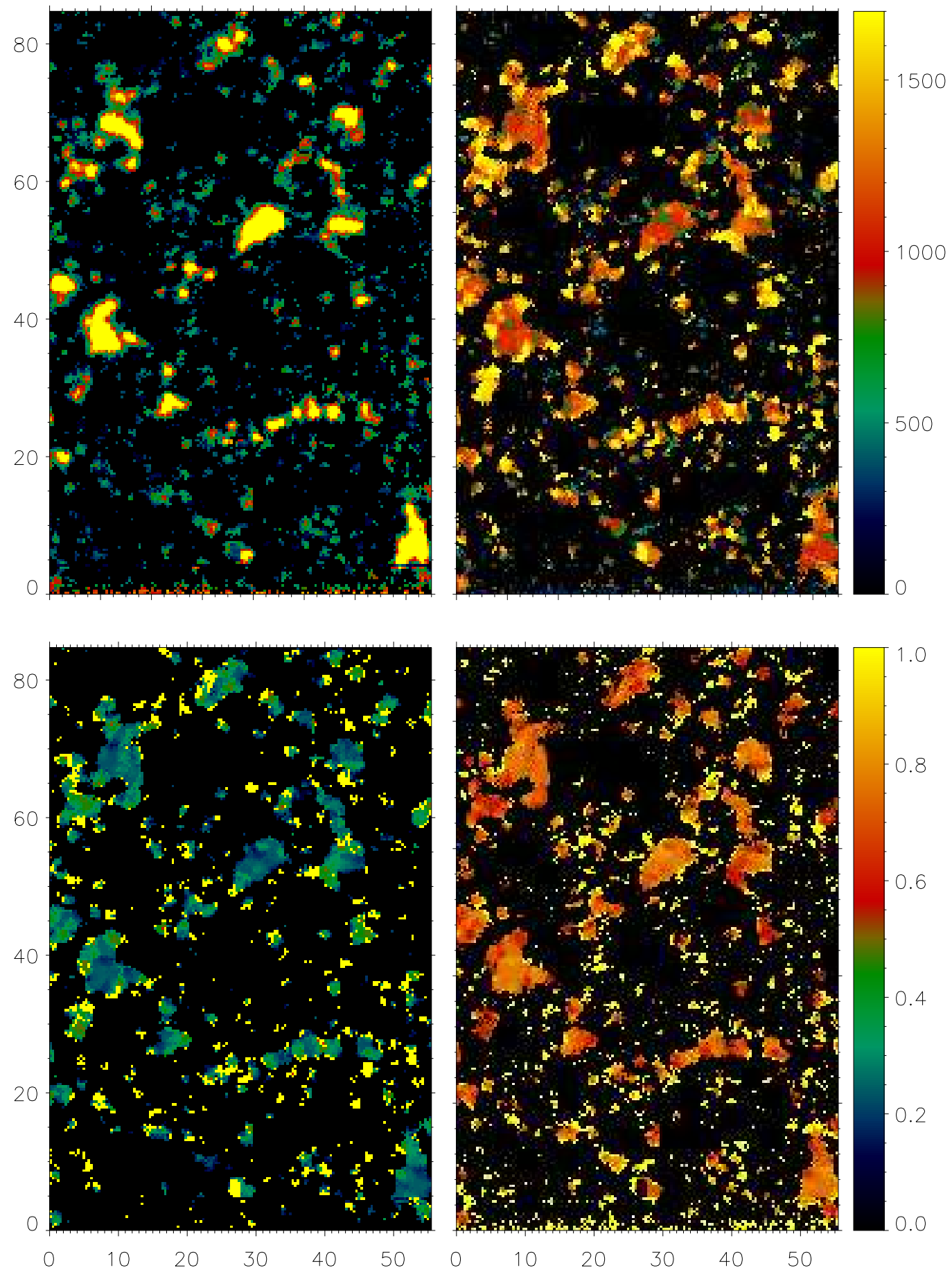

Fig. 2.- Map 1 (ASP). Upper left: Total magnetic filling factor, saturated at 0.10. The color scale in this panel ranges from 0 to 0.10 . Upper right: Field strength inferred from the $1 \mathrm{C}$ inversion $(\mathrm{G})$. Lower left: Relative filling factor of the strong field component. Lower right: Relative filling factor of the weak field component. In the lower left (right) image the color scale represents the percentage of the magnetic area occupied by strong (weak) fields. Spatial units are arc-seconds. Pixels appearing black in both images do not exhibit polarization signal above the noise and have been excluded from the analysis. 
Map 2
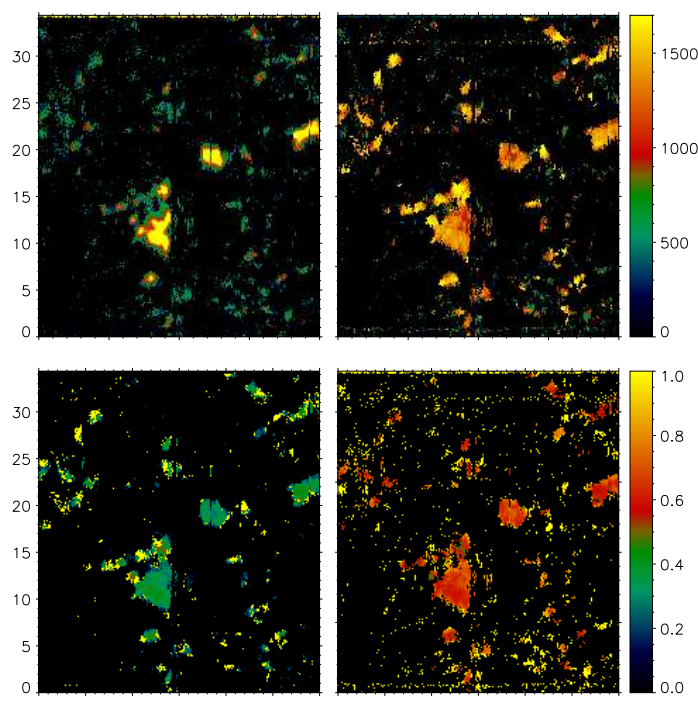

Map 3
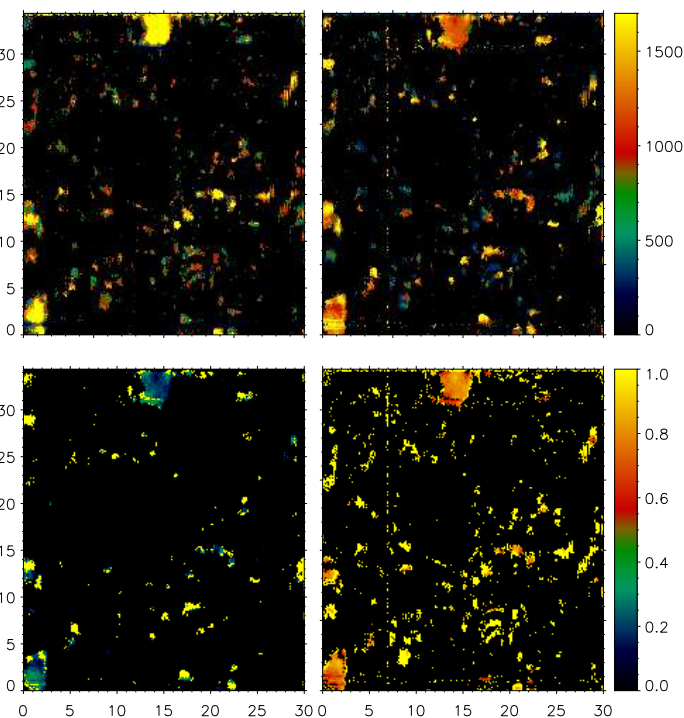

Fig. 3.- Maps 2 and 3 (DLSP). Four panels represent the following quantities for each map. Upper left: Total magnetic filling factor, saturated at 0.10 . The color scale in this panel ranges from 0 to 0.10 . Upper right: Field strength inferred from the $1 \mathrm{C}$ inversion $(\mathrm{G})$. Lower left: Relative filling factor of the strong field component. Lower right: Relative filling factor of the weak field component. In the lower left (right) image the color scale represents the percentage of the magnetic area occupied by strong (weak) fields. Spatial units are arcseconds. Pixels appearing black in both images do not exhibit polarization signal above the noise and have been excluded from the analysis. 
Table 1. Flux and mixed-strengths statistics

\begin{tabular}{|c|c|c|c|}
\hline & Map 1 & Map 2 & Map 3 \\
\hline Mean 1C flux $\left(\mathrm{Mx} \mathrm{cm}^{-2}\right)$ & 8.7 & 5.2 & 6.2 \\
\hline Mean 2C flux $\left(\mathrm{Mx} \mathrm{cm}^{-2}\right)$ & 9.2 & 5.4 & 6.1 \\
\hline Mean 1C field (G) & 1529 & 999 & 1064 \\
\hline Mean 2C field (G) & 1192 & 928 & 924 \\
\hline Pixels analyzed & $25.9 \%$ & $24.5 \%$ & $24.0 \%$ \\
\hline Pixels harboring & & & \\
\hline mixed strengths & $14.8 \%$ & $7.6 \%$ & $2.8 \%$ \\
\hline $\begin{array}{l}\text { Mixed strength in pixels } \\
\text { with } \alpha<0.1, B<500 \mathrm{G} \\
\text { Mean flux in }\end{array}$ & $0 \%$ & $0 \%$ & $0 \%$ \\
\hline strong fields $\left(\mathrm{Mx} \mathrm{cm}^{-2}\right)$ & 6.3 & 3.3 & 2.6 \\
\hline $\begin{array}{l}\text { Mean flux in } \\
\text { weak fields }\left(\mathrm{Mx} \mathrm{cm}^{-2}\right)\end{array}$ & 2.9 & 2.1 & 3.5 \\
\hline $\begin{array}{l}\text { Fraction of upflowing } \\
\text { area with strong fields }\end{array}$ & $12.9 \%$ & $1.6 \%$ & $9.5 \%$ \\
\hline $\begin{array}{l}\text { Fraction of downflowing } \\
\text { area with strong fields }\end{array}$ & $28.2 \%$ & $24.4 \%$ & $13.2 \%$ \\
\hline
\end{tabular}




\section{Reliability of the results}

We have not been concerned thus far with problems such as line asymmetries or details of the sub-pixel field distribution. This would be a concern if we were interested in obtaining a detailed description of the solar atmosphere, including velocity and magnetic field gradients. Our goal, however, is not that, but rather to demonstrate that weak and strong fields generally coexist at sub-arcsecond spatial scales. How is this overall conclusion affected by the simplifying assumptions employed in our work? This important question is explored in the present section by means of numerical simulations. Before going into the details, though, it is probably a good idea to consider the broader context of the subject in order to put our work into perspective.

In doing analysis of solar magnetic fields based on spectro-polarimetric observations there are various levels of complexity that one can consider, depending on the sophistication of the physical models employed. The most simplistic analyses are the "traditional" techniques, such as the line ratio, the separation of Stokes V extrema, or fits by Gaussian profiles. These techniques do not involve radiative transfer calculations and do not require a detailed knowledge of the atmospheric model. They typically provide only a rough approximation to the magnetic field, but are unable to deal with line asymmetries due to unresolved inhomogeneities, atmospheric gradients, etc. The most sophisticated techniques make use of full radiative transfer calculations in detailed model atmospheres to fit the observed profiles in a least-squares sense (inversion codes).

Most of the published work (see references in $§ 1$ ) makes use of the simplistic techniques to infer "typical" values of the photospheric field in the quiet Sun. These simplified analyses led to the inference that network fields are typically strong $(\simeq 1.5 \mathrm{kG})$, or that internetwork fields appear to be mostly $\mathrm{kG}$ when observed in the visible and sub-kG $(\simeq 300 \mathrm{G})$ when observed in the infrared. Very few authors (e.g., the works of Sánchez Almeida \& Lites 2000; Socas-Navarro \& Sánchez Almeida 2002) have used inversions that consider detailed models with atmospheric gradients. The overall picture (i.e., whether the fields are weak or strong) does not change signifincantly, although the inversion codes obviously provide more information. Detailed inversions have not been carried out in the infrared so far. The analyses published in the literature are derived from fits of Gaussian functions to the observed profiles.

Our analysis in this paper lies somewhere between the two ends of the range of sophistication. We calculate actual Stokes profiles from a model atmosphere, but do not consider line-of-sight variations of the quantities. The thermodynamics of our models is probably reasonably good, as suggested by the fits shown in Fig 1. However, there are two important simplifications. First, we do not consider line-of-sight gradients of the field. Second, 
we parameterize the unresolved field distribution by two discrete field values of $300 \mathrm{G}$ and $1700 \mathrm{G}$. It is important to note that none of these assumptions are worse than those of the traditional methods (save for the exceptions noted above) which are based on single-valued fields without height variations.

We have carried out some numerical simulations in order to gain insights into the adequateness of our approximations. First consider how our two-point approach is representative of the sub-pixel field distribution. Suppose that we have an unresolved probability distribution function (PDF) of field strengths $f(B)$. The filling factor of fields with a strength between $B$ and $B+\Delta B$ is:

$$
\alpha(B, B+\Delta B)=\int_{B}^{B+\Delta B} f(B) d B
$$

The emerging profile $P(\lambda)$ from such distribution is:

$$
P(\lambda)=\int_{0}^{\infty} f(B) P_{B}(\lambda) d B
$$

where $P_{B}(\lambda)$ denotes the profile produced by a field of strength $B$. For the moment we are only interested in horizontal inhomogeneities. The effects of gradients of $B$ along the line of sight will be discussed later. If we consider $P(\lambda)$ as a simulated observation and apply to it the procedure introduced in $\S 2.2$ above, we can compare the inferred values with the actual PDF, $f(B)$, employed. Table 2 shows the results of various tests with different shapes of the PDF. As proposed by Emonet \& Cattaneo (2001), and by Khomenko et al. (2003), Socas-Navarro \& Sánchez Almeida (2003), and Trujillo Bueno et al. (2004), we adopted an exponential dependence ${ }^{2}$ for $f(B)$ with a normalization factor $N^{-1}=\int_{0}^{\infty} f(B) d B$. The filling factor of fields stronger than $2 \mathrm{kG}$ has been set to zero. We also considered a Diracdelta $\operatorname{PDF}\left(\delta\left(B-B_{0}\right)\right)$, which simply means that the field is homogeneous over the resolution element, with a strength $B_{0}$.

The results in Table 2 suggest that the filling factors that we obtained for $B=300 \mathrm{G}$ and $B=1700 \mathrm{G}$ are more or less representative of the weak and strong fields present in more complex distributions expected to be present in the actual quiet Sun. We also tested the sensitivity of our results to the values that we used to represent weak and strong fields.

\footnotetext{
${ }^{2}$ It should be noted that the PDF obtained by Emonet \& Cattaneo (2001) from the simulations is actually somewhat more complicated. Their PDF is a stretched exponential in the absence of net unsigned flux, and has a shoulder when such flux is non-zero.
} 
To this aim we repeated the calculations above but this time using 500 and $1500 \mathrm{G}$. The results obtained, listed in Table 3, are very similar to those of Table 2.

Let us now consider the issue of velocity and magnetic field gradients along the line of sight. Such gradients give rise to asymmetries in the line profiles. We introduced gradients in our simulated observations and tested their effects on the filling factors inferred by our analysis. The line-of-sight velocity that we introduced varies linearly between $\tau_{500}=10$ and $\tau_{500}=10^{-3}$ (with $\tau_{500}$ denoting the optical depth at $500 \mathrm{~nm}$ ). The amplitude of the variation is of $2 \mathrm{~km} \mathrm{~s}^{-1}$. The field strength varies linearly as well, from $B$ at $\tau_{500}=10^{-1}$ (with $B$ ranging from 0 to $2 \mathrm{kG}$ ) to zero at $\tau_{500}=10^{-3}$. The asymmetric profiles calculated from the PDFs above are again taken as simulated observations and applied our analysis (which neglects gradients). The results from this experiment are summarized in Table 4 (the numbers within parentheses are obtained when the velocity gradient is doubled). These values indicate that the presence of asymmetries does not invalidate our conclusions on the coexistence of field strengths in the resolution element.

\section{Conclusions}

This paper reports on observational evidence for mixed field strengths in the quiet Sun $(\sim 300$ and $\sim 1700 \mathrm{G})$ on spatial scales smaller than 0."6. Mixed strengths are found in network and internetwork magnetic elements. The results for the internetwork are not entirely unexpected. Socas-Navarro \& Sánchez Almeida (2003) proposed that such mixture is the most natural explanation for the discrepancy between visible and infrared observations. Our work strongly supports their conjecture and starts to bridge the gap between the two types of observations. The presence of weak fields inside network patches is more surprising and had not been anticipated before.

The reliability of our results is backed by several facts. Our 1C analysis reproduces what had been obtained in the past from visible spectro-polarimetric observations. When we extend the analysis to $2 \mathrm{C}$ we find that the merit function $\chi^{2}$ is systematically smaller, even though we did not add free parameters in the model. Therefore, our $2 \mathrm{C}$ analysis is at least as reliable as comparable $1 \mathrm{C}$ studies which have been used thus far. In order to ensure a conservative criterion for the occurrence of $2 \mathrm{C}$, our procedure was implemented with a "preference" for $1 \mathrm{C}$ solutions when possible. The spatial distributions of the filling factors $\alpha_{w}$ and $\alpha_{s}$ are smooth, exhibiting spatial coherence even though each pixel has been inverted separately. These distributions, as well as the other results obtained from our study, are consistent in the three maps analyzed (which have been obtained from two different instruments). Several numerical tests (§4) show that our method is able to distinguish 
Table 2. Results from simulations. Inferrences using values of $300 \mathrm{G}$ and $1700 \mathrm{G}$.

\begin{tabular}{ccccc}
\hline \hline $\begin{array}{c}\text { PDF } \\
(B \text { in } \mathrm{G})\end{array}$ & $\begin{array}{c}\text { Actual } \\
\alpha(B<1 \mathrm{kG})\end{array}$ & $\begin{array}{c}\text { Inferred } \\
\alpha(B=300 \mathrm{G})\end{array}$ & $\begin{array}{c}\text { Actual } \\
\alpha(B>1 \mathrm{kG})\end{array}$ & $\begin{array}{c}\text { Inferred } \\
\alpha(B=1.7 \mathrm{kG})\end{array}$ \\
\hline$f=\delta(B-100)$ & 1.00 & 1.00 & 0.00 & 0.00 \\
$f=\delta(B-2000)$ & 0.00 & 0.00 & 1.00 & 1.00 \\
$f=N \exp (-B / 100)$ & 1.00 & 1.00 & 0.00 & 0.00 \\
$f=N \exp (-B / 300)$ & 0.96 & 0.93 & 0.04 & 0.07 \\
$f=N \exp (-B / 600)$ & 0.84 & 0.82 & 0.16 & 0.18 \\
$f=N \exp (-B / 1000)$ & 0.73 & 0.75 & 0.27 & 0.25 \\
$f=N \exp (-B / 1500)$ & 0.66 & 0.70 & 0.34 & 0.30 \\
$f=N \exp (-B / 2000)$ & 0.62 & 0.67 & 0.38 & 0.33 \\
\hline
\end{tabular}

Table 3. Results from simulations. Inferrences using values of $500 \mathrm{G}$ and $1500 \mathrm{G}$.

\begin{tabular}{ccccc}
\hline \hline PDF & $\begin{array}{c}\text { Actual } \\
(B \text { in } \mathrm{G})\end{array}$ & $\begin{array}{c}\text { Inferred } \\
\alpha(B<1 \mathrm{kG})\end{array}$ & $\begin{array}{c}\text { Actual } \\
\alpha(B=500 \mathrm{G})\end{array}$ & $\begin{array}{c}\text { Inferred } \\
(B>1 \mathrm{kG})\end{array}$ \\
$\alpha(B=1.5 \mathrm{kG})$ \\
\hline$f=\delta(B-100)$ & 1.00 & 1.00 & 0.00 & 0.00 \\
$f=\delta(B-2000)$ & 0.00 & 0.00 & 1.00 & 1.00 \\
$f=N \exp (-B / 100)$ & 1.00 & 1.00 & 0.00 & 0.00 \\
$f=N \exp (-B / 300)$ & 0.96 & 0.97 & 0.04 & 0.03 \\
$f=N \exp (-B / 600)$ & 0.84 & 0.79 & 0.16 & 0.21 \\
$f=N \exp (-B / 1000)$ & 0.73 & 0.77 & 0.27 & 0.33 \\
$f=N \exp (-B / 1500)$ & 0.66 & 0.60 & 0.34 & 0.40 \\
$f=N \exp (-B / 2000)$ & 0.62 & 0.57 & 0.38 & 0.43 \\
\hline
\end{tabular}


between the filling factors of weak and strong fields in the presence of unresolved PDFs and/or line asymmetries. Finally, our results are sensible from a physical point of view. For example, we find mixed strengths in internetwork locations showing strong $1 \mathrm{C}$ fields but not in those showing weak $1 \mathrm{C}$ fields. Moreover, the relative fractional area occupied by weak and strong fields is different for upflows and downflows, with the downflows having a larger filling factor of $\mathrm{kG}$ fields (in agreement with existing simulations).

It is important to keep in mind that the mixed strengths that we have detected may be just the tip of the iceberg. It is very likely that we only see the most conspicuous ones. We have chosen to parameterize the sub-pixel field distribution by two discrete values at 300 and 1700 G. However, we know from both observations and simulations that quiet Sun fields obey a continuous probability distribution, with the weaker fields covering a larger fraction of the resolution element. Unfortunately, according to Socas-Navarro (2004b), we can only infer (at most) two points of such distribution using visible observations. Simultaneous visible and infrared observations will offer a much more detailed picture. It might then be possible to infer more than two points of the distribution.

\section{REFERENCES}

Cattaneo, F. 1999, ApJ, 515, L39

Domínguez Cerdeña, I., Kneer, F., \& Sánchez Almeida, J. 2003a, ApJ, 582, L55

Domínguez Cerdeña, I., Sánchez Almeida, J., \& Kneer, F. 2003b, A\&A, 407, 741

Emonet, T., \& Cattaneo, F. 2001, ApJ, 560, L197

Grossmann-Doerth, U., Keller, C. U., \& Schüssler, M. 1996, A\&A, 315, 610

Keller, C. U., Deubner, F.-L., Egger, U., Fleck, B., \& Povel, H. P. 1994, A\&A, 286, 626

Khomenko, E. V., Collados, M., Solanki, S. K., Lagg, A., \& Trujillo Bueno, J. 2003, A\&A, 408, 1115

Lin, H. 1995, ApJ, 446, 421

Lin, H., \& Rimmele, T. 1999, ApJ, 514, 448

Lites, B. W. 1996, Solar Phys., 163, 223

—. 2002, ApJ, 573, 431 
Lites, B. W., \& Socas-Navarro, H. 2004, ApJ, in press

Sánchez Almeida, J., Emonet, T., \& Cattaneo, F. 2003, ApJ, 585, 536

Sánchez Almeida, J., \& Lites, B. W. 2000, ApJ, 532, 1215

Schüssler, M. 2003, in ASP Conf. Ser. (San Francisco: ASP), Vol. 307, Solar Polarization Workshop 3, ed. J. Trujillo-Bueno \& J. Sánchez Almeida, 601

Socas-Navarro, H. 2001, in ASP Conf. Ser. 236: Advanced Solar Polarimetry - Theory, Observation, and Instrumentation, 487

Socas-Navarro, H. 2004a, ApJ, in press

-. 2004b, ApJ, in press

Socas-Navarro, H., Martínez Pillet, V., \& Lites, B. W. 2004, ApJ, in press

Socas-Navarro, H., \& Sánchez Almeida, J. 2002, ApJ, 565, 1323

-. 2003, ApJ, 593, 581

Stein, R. F., \& Nordlund, A. 2002, in SOLMAG2002 IAU Colloquium 188, Sawaya-Lacoste (ed.), ESA SP-505, 83

Trujillo Bueno, J., Schukina, N., \& Asensio Ramos, A. 2004, Nature, in press 
Table 4. Results from simulations with asymmetric profiles.

\begin{tabular}{ccccc}
\hline \hline PDF & $\begin{array}{c}\text { Actual } \\
(B \text { in } \mathrm{G})\end{array}$ & $\begin{array}{c}\text { Inferred } \\
\alpha(B<1 \mathrm{kG})\end{array}$ & $\begin{array}{c}\text { Actual } \\
\alpha(B=500 \mathrm{G})\end{array}$ & $\begin{array}{c}\text { Inferred } \\
\alpha(B>1 \mathrm{kG})\end{array}$ \\
$\alpha(B=1.5 \mathrm{kG})$ \\
\hline$f=\delta(B-100)$ & 1.00 & $1.00(0.97)$ & 0.00 & $0.00(0.03)$ \\
$f=\delta(B-2000)$ & 0.00 & $0.01(0.00)$ & 1.00 & $0.99(1.00)$ \\
$f=N \exp (-B / 100)$ & 1.00 & $0.99(0.95)$ & 0.00 & $0.01(0.05)$ \\
$f=N \exp (-B / 300)$ & 0.96 & $0.91(0.87)$ & 0.04 & $0.09(0.13)$ \\
$f=N \exp (-B / 600)$ & 0.84 & $0.80(0.75)$ & 0.16 & $0.20(0.25)$ \\
$f=N \exp (-B / 1000)$ & 0.73 & $0.73(0.75)$ & 0.27 & $0.27(0.25)$ \\
$f=N \exp (-B / 1500)$ & 0.66 & $0.69(0.63)$ & 0.34 & $0.31(0.37)$ \\
$f=N \exp (-B / 2000)$ & 0.62 & $0.66(0.60)$ & 0.38 & $0.34(0.40)$ \\
\hline
\end{tabular}

